Philosophie ANTIQUE
Philosophie antique

Problèmes, Renaissances, Usages

$14 \mid 2014$

Le devoir

\title{
Katja Maria VogT, Belief and Truth. A Skeptic Reading of Plato
}

\section{Thomas Bénatouill}

\section{OpenEdition}

\section{Journals}

Édition électronique

URL : https://journals.openedition.org/philosant/847

DOI : 10.4000/philosant.847

ISSN : 2648-2789

\section{Éditeur}

Éditions Vrin

\section{Édition imprimée}

Date de publication : 1 novembre 2014

Pagination : 338-342

ISBN : 978-2-7574-0855-1

ISSN : $1634-4561$

Référence électronique

Thomas Bénatouill, « Katja Maria vogt, Belief and Truth. A Skeptic Reading of Plato », Philosophie antique [En ligne], 14 | 2014, mis en ligne le 01 novembre 2018, consulté le 03 décembre 2022. URL : http:// journals.openedition.org/philosant/847 ; DOI : https://doi.org/10.4000/philosant.847

\section{(ब) $(\oplus \Theta$}

Creative Commons - Attribution - Pas d'Utilisation Commerciale - Pas de Modification 4.0 International - CC BY-NC-ND 4.0

https://creativecommons.org/licenses/by-nc-nd/4.0/ 
laisser de côté ces notions, au moins dans un premier temps, pour mieux saisir en quelque sorte l'aporie à l'état brut, conformément à l'esprit de l'œuvre ? L'étude de F. Teisserenc, si elle tend à complexifier le problème, présente cependant un intérêt majeur : elle fait apparaître une faiblesse dans la thèse que l'on retient traditionnellement, thèse qui parvient à sa meilleure formulation avec Sedley, selon laquelle la notion de Forme en tant que non-dit du Théétète serait de nature à résoudre l'aporie finale. Or tel n'est pas le cas, selon F. Teisserenc. Ainsi le passage 146e qui pose le problème de savoir ce que l'episteme peut bien être en soi (gnonai epistemen auto hoti pot'estin) appellerait d'emblée une solution négative, car il n'y a pas d'en-soi de la science. F. Teisserenc montre ainsi que l'aporie finale ne résulte pas d'un simple changement de perspective (thèse de Rowe), qu'elle reste fondamentale et que sa solution réside moins dans la Forme que dans la notion de puissance (dynamis) (thèse de M. Dixsaut). Il faut dès lors se rapporter à République IV et V, notamment avec la dualité entre les pros alla et les auta kath'hauta - distinction qui débouche avec les pros alla sur la notion de dynamis. On sait d'ailleurs - F. Teisserenc aurait pu le rappeler - que cette dualité est très importante dans le système platonicien des agrapha dogmata. Force est donc de constater que cette solution dépasse la question originairement socratique du ti estin, ramenant naïvement la science à une essence. Teisserenc remarque alors d'une manière très subtile que le passage mathématique sur les puissances (dynameis) devait faire signe en direction de la solution, précisément la science comme puissance.

Il ne nous est pas possible, dans le cadre de ce compte rendu, de faire le point sur toutes les études. Aussi ne pouvons-nous qu'évoquer la seconde partie de l'ouvrage qui traite de l'importante postérité du Théétète. Sont examinés successivement les différentes versions du dialogue qui ont pu circuler dans l'antiquité (H. Tarrant), le rapport au stö̈cisme (A. Macé), la place du dialogue dans la classification de Thrasylle (D. Sedley), «l'unitarisme » de l'Anonyme médio-platonicien (M. Bonazzi), le rapport au plotinisme (S. Magrin) et à Montaigne (B. Sève). Ces études, toutes aussi passionnantes les unes que les autres, se prêtent cependant moins à la discussion. Ce qui a retenu spécialement notre attention dans cet ouvrage - qui fera certainement date dans les études sur le Théétète - réside dans son examen approfondi des questions relatives au caractère à la fois maïeutique et aporétique de ce grand dialogue.

Jean-Luc PÉRILLIÉ

Université Paul Valéry-Montpellier 3

Katja Maria Vogt, Belief and Truth. A Skeptic Reading of Plato, Oxford University Press, 2012, ix-209 pages, ISBN : 976-0-19-991681-8.

Cet ouvrage cherche à mettre en lumière et reconstituer une manière antique négligée d'analyser la connaissance que l'auteure désigne comme « socratique » (p. 3), comme «pyrrhonienne» (p. 184) ou comme une «lecture sceptique de Platon », et dont elle trouve et analyse les principaux éléments chez Platon, Sextus Empiricus et les stö̈ciens. Cette lignée est définie comme liant immédiatement les questions épistémologiques à des questions normatives à propos de notre incapacité d'examiner nos propres idées du fait de nos préférences (p. 3). L'une de ses thèses caractéristiques, selon Katja Vogt (désormais KV), est que la croyance (belief 
$=(o x a)^{1}$ est une « attitude cognitive déficiente » si bien qu'il existe une hétérogénéité entre croyance et connaissance et que la seconde doit remplacer la première (et non la corriger ou la compléter). Qu'une hiérarchie forte entre doxa et connaissance soit défendue par Socrate, Platon, les stoïciens et Sextus Empiricus est assez connu, même s'il est rare de trouver une approche transversale de cette question ${ }^{2}$. Mais qu'il existe des analyses épistémologiques communes à ces philosophes, voire qu'ils s'inspirent les uns des autres, est une thèse beaucoup plus forte. En outre, comme l'épistémologie contemporaine a largement tendance à tenir la connaissance pour un type de croyance, l'étude de différentes versions antiques de la thèse contraire a un enjeu non seulement historique mais aussi philosophique et critique.

L'ouvrage commence par un chapitre sur le thème classique de l'ignorance comme croyance que l'on sait (presumed knowledge) chez Platon, en particulier dans les premiers dialogues, mais elle en propose une analyse originale parce qu'elle se penche sur l'Ion et le Philèbe (plutôt que sur le Gorgias ou le Ménon) et parce qu'elle insiste sur la dimension pour ainsi dire psychologique de ce phénomène : ce que Socrate montrerait, c'est que l'ignorance épistémologiquement répréhensible résulte d'une surestimation de nous-mêmes que nous « transférons » à nos capacités cognitives si bien que nous prétendons avec trop de confiance savoir des choses (sur de nombreux sujets autres que nous-mêmes) que nous avons souvent simplement apprises d'autres personnes et que nous ne comprenons pas (p. 26-30). La surestimation de soi, au principe de cette transferred ignorance, peut être liée à une certaine position sociale ou à la détention d'une compétence spécialisée, comme le montre l'enquête que Socrate raconte dans l'Apologie, et ne peut être évitée qu'en devenant conscient de l'impuissance de l'homme à atteindre une sagesse véritable, indépendante de ses facultés limitées de connaître : il doit se contenter d'une « sagesse humaine » à propos des questions les plus importantes.

L'Ion confirmerait que ce sont les intellectuels qui sont les plus susceptibles de faire preuve de transferred ignorance et la manière dont Ion s'approprie les idées d'Homère qu'il récite illustrerait en particulier le fait que nous empruntons souvent nos idées à d'autres personnes sans les comprendre, si bien que nous ne pouvons pas les défendre, alors que Socrate est capable de distinguer ses propres idées de celles des autres. Cette analyse est très intéressante, mais il n'est pas certain que, pour Platon, la source véritable de l'ignorance inconsciente d'elle-même soit l'incapacité de faire cette distinction ou même une surestimation de nous-mêmes

1. KV (p. 10) refuse la traduction « opinion » parce qu'elle suggère que la doxa est incluse dans une catégorie plus large, la «croyance », qui serait exempte des défauts de l'opinion. Selon l'épistémologie socratique, la doxa est au contraire la croyance au sens large de tenir quelque chose pour vrai.

2. Pour une approche parallèle passant par d'autres auteurs, voir C. Lévy, « Le concept de doxa des Stoïciens à Philon d'Alexandrie : essai d'étude diachronique », dans J. Brunschwig et M. C. Nussbaum, Passions and Perceptions. Studies in Hellenistic Philosophy of Mind, Paris/Cambridge, Editions MSH/Cambridge University Press, 1993, p. 250-284. KV cite très peu d'études en français ou en italien. Elle ne mentionne par exemple pas non plus l'ouvrage classique d'Y. Lafrance, La théorie platonicienne de la Doxa, Montréal/Paris, Bellarmin/Les Belles-Lettres, 1981. 
(favorisée par notre statut social). Il est remarquable que la première objection contre les prétentions au savoir d'Ion est la même que celle formulée à l'égard de la rhétorique de Gorgias : ses compétences techniques n'ont pas d'objet déterminé et ne sont donc pas définissables (Ion, 537d et Gorgias, $449 \mathrm{~d}$ sqq.). Le principal problème semble donc être lié au fait que le rhapsode, l'orateur ou le sophiste se croit compétent sur toutes les affaires ou choses que peut évoquer son discours, comme si ces dernières ne possédaient pas une autonomie et des spécificités réclamant pour chacune d'entre elles - une compétence technique propre et limitée. L'ignorance propre aux intellectuels est moins «transférée » de leur image prétentieuse d'eux-mêmes à une (illusoire) connaissance des autres choses que de leur maîtrise du discours sur tous les sujets à une prétendue connaissance des objets du discours.

Cette question du rapport entre compétence et objet est justement au cœur du second chapitre, qui porte sur le statut de la doxa dans la République. KV reprend un débat désormais classique : croyance et connaissance ont-elles chacune leur objet propre (si bien qu'on ne peut avoir de croyance sur les Formes intelligibles ni de connaissance des particuliers sensibles) ou, comme l'a soutenu en particulier Gail Fine, consistent-elles en propositions de type différent, qui peuvent éventuellement porter sur les mêmes objets ? KV montre bien pourquoi ces deux interprétations sont insatisfaisantes et soutient pour sa part que, pour Platon, croyance et connaissance sont adaptées ou destinées à certains objets, mais qu'elles peuvent néanmoins s'appliquer de manière déficiente à d'autres objets (comme dans le cas d'une croyance à propos du bien). Elle souligne en outre la notion de « croyance sans connaissance » utilisée par Socrate (506c6-8).

Dans le troisième chapitre, sur le Théétète, KV se concentre sur la seconde partie et suggère que son incapacité de définir la croyance fausse résulterait du présupposé initial (187c) selon lequel il existe deux types de croyance, la vraie et la fausse. Toutes les croyances seraient en réalité du même type dans la mesure où elles sont toutes aussi déficientes par rapport à la connaissance (p. 81). KV contribue donc de manière intéressante à un courant d'interprétation du Théétète selon lequel le dialogue montre que l'on ne peut pas transformer la croyance, même vraie, en connaissance. La différence entre croyance vraie et fausse ne serait établie que de l'extérieur, celui qui possède une croyance vraie ne pouvant jamais savoir (à partir d'elle) qu'elle est vraie. Ce constat serait également à la base de la conception stoïcienne de la doxa (présentée plus en détail dans le dernier chapitre), qui pourrait de ce fait avoir été élaborée à partir d'une lecture de la seconde partie du Théétète (p. 94). La vérité serait ainsi nécessairement liée à la connaissance. La thèse peut être soutenue, mais on aurait aimé savoir pourquoi, dans ces conditions, Platon maintient la pertinence de la distinction entre opinion fausse et opinion vraie au moins dans certains dialogues comme la fin du Ménon ou le Timée ${ }^{1}$.

1. De manière analogue, dans la conception stoïcienne de la doxa, il existe - comme le montre bien $\mathrm{KV}$ - deux types de croyances, l'assentiment à une représentation non cataleptique et l'assentiment faible à une représentation cataleptique, la seconde représentant un progrès (p. 163-164 et 170), même si elle n'est pas vraie. Tout en étant considérée comme toujours mauvaise, la doxa comprend donc différents niveaux et offre ainsi un passage vers son dépassement et la constitution de la science. Est-ce que l'opinion ne pourrait pas avoir 
Les trois chapitres suivants portent sur Sextus Empiricus ${ }^{1}$. KV propose dans le premier de lire le pyrrhonisme dans le prolongement du Théétète. L'analyse est convaincante, mais soulève un problème plus général. Pourquoi en passer ici par Sextus alors qu'il semblerait beaucoup plus pertinent de s'intéresser à la Nouvelle Académie d'Arcésilas et de Carnéade ? KV fait de temps en temps allusion au scepticisme académicien, mais considère que les questions qui l'intéressent sont traitées plus explicitement par les pyrrhoniens (p. 10). Pourtant, la différence entre opinion et connaissance qui fait l'objet des chapitres 1 à 3 et 8 est tout à fait importante pour la Nouvelle Académie : Arcésilas aurait précisément trouvé son point de départ dans la thèse de Zénon, évidemment inspirée de Socrate, selon laquelle le sage ne doit pas avoir de croyance (Cicéron, Luc. 76-77). La Nouvelle Académie aurait donc permis d'établir une continuité beaucoup plus plausible historiquement entre Socrate, Platon (et en particulier le Théétète), une forme de scepticisme et le stoïcisme. Mais la Nouvelle Académie incarne aussi les problèmes d'une continuité de ce type, puisqu'elle a cherché à montrer l'infidélité du stoïcisme à l'inspiration socratique et platonicienne, dénonçant son dogmatisme au moyen des arguments de Socrate contre les sophistes ${ }^{2}$. En outre, le souci de Sextus (et d'Énésidème) de se distinguer nettement de la Nouvelle Académie permettrait de montrer aussi que l'idée d'une inspiration socratico-platonicienne du pyrrhonisme est peu plausible, contrairement à ce que suggère $\mathrm{KV}$ (p. 121-122) 3. $^{\mathrm{A}}$ la fois argument et objection, la Nouvelle Académie semble ainsi incontournable pour toute reconstitution d'une épistémologie socratique ou d'une lecture sceptique de Platon fondées sur une hétérogénéité forte entre doxa et connaissance.

Dans les deux autres chapitres sur Sextus, sont évoqués les buts de la recherche sceptique et l'objection selon laquelle les sceptiques ne peuvent penser s'ils refusent toute croyance ${ }^{4}$. La première question et en particulier le problème de savoir comment Sextus peut dire que le sceptique recherche la vérité ont été beaucoup

un statut similaire chez Platon ? C'est d'ailleurs ce que suggère KV dans son chapitre sur la République mais qu'elle ne semble pas maintenir dans celui sur le Théétète.

1. Deux d'entre eux sont déjà parus dans des versions différentes, comme d'ailleurs le chapitre sur la République.

2. Voir par exemple Cicéron, Luc. 91 et Div. II, 9-14 pour une réutilisation par Carnéade contre la dialectique et la divination stoïciennes de l'argument du Gorgias ou de l'Ion mentionné plus haut.

3. En revanche, il est tout à fait juste que la première partie du Théétète et son analyse du relativisme et du désaccord permettent d'éclairer les motivations du scepticisme de Pyrrhon, d'Énésidème et de Sextus, comme le propose KV dans son chapitre 4. Lorsqu'elle parle de « lecture » stoïcienne ou sceptique de Platon, KV suggère en fait des rapprochements entre doctrines (telles qu'elle les interprète) plutôt qu'elle n'essaye d'établir des lectures antiques ou influences effectives à partir de la comparaison précise de textes.

4. Sur ce problème difficile et important, qui concerne $P H$ II, 1-11 et $A M$ VIII, 337 336a et l'usage des concepts par le sceptique, KV propose une critique juste de la lecture reçue de ces passages et une interprétation nettement meilleure (renvoyant au contexte dialectique et à l'invocation de la nature de l'âme en $P H$ I, 24 : voir note 11 ci-dessous). Il est juste dommage qu'elle ne discute pas l'analyse détaillée de ce problème et de ces passages proposée par L. Corti, Scepticisme et langage, Paris, Vrin, 2009, p. 185-206. 
discutés récemment ${ }^{1} ; \mathrm{KV}$ propose une solution selon laquelle le scepticisme partage certaines exigences cognitives avec les dogmatiques et chercherait avant tout à se prémunir de la tendance de l'esprit à donner trop facilement son assentiment : il s'agirait d'élaborer des techniques fiables pour éviter les erreurs, ce qui est plus important que de tenir quelque chose pour vrai. Cette solution me semble tout à fait judicieuse comme réponse au problème posé, mais elle me semble caractériser la position néoacadémicienne plutôt que celle de Sextus qui, à ma connaissance, n'attribue pas à l'âme humaine une tendance spontanée à donner trop rapidement son assentiment ${ }^{2}$.

Reste le dernier chapitre sur le stoïcisme, qui est sans doute le plus intéressant, à cause de l'originalité de la thèse soutenue et de la rigueur de sa démonstration. $\mathrm{KV}$ montre que, contrairement à une lecture fréquente aujourd'hui, une croyance n'est jamais vraie pour les stoïciens : seules les assertions ou propositions peuvent être vraies et seul celui qui sait est en possession de la vérité. Pour le montrer, KV analyse avec précision les différentes caractéristiques de la doxa chez les stoïciens, mais aussi la distinction connue entre la vérité et le vrai, leur conception de la science et la définition des émotions comme un certain type d'opinions. Le seul inconvénient de l'interprétation de KV est qu'elle n'explique pas bien pourquoi l'opinion est définie comme un assentiment « faible » mais aussi « faux $»^{3}$, et l'on se demande donc si KV ne devrait pas envisager plus sérieusement l'hypothèse (rejetée p. 171 au nom du principe de bivalence) que l'opinion pourrait être tenue pour fausse mais jamais pour vraie, même si, au sens strict, seuls les axiomata sont vrais ou faux.

L'ouvrage de KV propose donc des hypothèses très suggestives et souvent convaincantes à propos du statut de l'opinion, de la connaissance ou de la recherche de la vérité chez Platon, Sextus Empiricus et les stoïciens. En revanche, malgré ma sympathie, voire mon adhésion à l'égard de ses thèses plus générales, selon lesquelles ces auteurs formeraient une famille épistémologique « socratique » et proposeraient une « lecture sceptique de Platon », il me semble que leur démonstration demeure incomplète.

Thomas BÉNATOUÏL

Université Charles de Gaulle-Lille 3

1. KV estime que les contemporains des sceptiques antiques n'ont pas soulevé l'objection selon laquelle les techniques d'argumentation des sceptiques montrent qu'ils ne cherchent pas la vérité (mais la suspension en tant que telle). Mais l'objection peut être trouvée dans des textes comme Cicéron, Luc. 60 ou Epictète, Entretiens, II, 20, 21, qui visent les Académiciens.

2. C'est un reproche qu'il fait avant tout à ses adversaires dogmatiques, si bien qu'il ne présente pas son scepticisme comme une discipline imposée à l'âme contre ses instincts naturels (il invoque au contraire ces derniers en faveur du scepticisme, comme le montre bien KV p. 154-156 à propos de $P H \mathrm{I}, 24)$. Voir au contraire $L u c .108$ pour la description par Clitomaque de Carnéade comme un Hercule terrassant l'hydre de l'assentiment.

3. KV estime que faux n'indique que la déficience liée à la faiblesse de l'assentiment (p. 164), ce qui est assez peu vraisemblable dans ce contexte. 